\title{
Saturated Fatty Acids and Risk of Coronary Heart Disease: Modulation by Replacement Nutrients
}

\author{
Patty W. Siri-Tarino • Qi Sun • Frank B. Hu • \\ Ronald M. Krauss
}

Published online: 14 August 2010

(C) The Author(s) 2010. This article is published with open access at Springerlink.com

\begin{abstract}
Despite the well-established observation that substitution of saturated fats for carbohydrates or unsaturated fats increases low-density lipoprotein (LDL) cholesterol in humans and animal models, the relationship of saturated fat intake to risk for atherosclerotic cardiovascular disease in humans remains controversial. A critical question is what macronutrient should be used to replace saturated fat. Substituting polyunsaturated fat for saturated fat reduces LDL cholesterol and the total cholesterol to high-density lipoprotein cholesterol ratio. However, replacement of saturated fat by carbohydrates, particularly refined carbohydrates and added sugars, increases levels of triglyceride and small LDL particles and reduces high-density lipoprotein cholesterol, effects that are of particular concern in the context of the increased prevalence of obesity and insulin resistance. Epidemiologic studies and
\end{abstract}

P. W. Siri-Tarino $\cdot$ R. M. Krauss $(\bowtie)$

Atherosclerosis Research,

Children's Hospital Oakland Research Institute,

5700 Martin Luther King Junior Way,

Oakland, CA 94609, USA

e-mail: rkrauss@chori.org

P. W. Siri-Tarino

e-mail: psiri@chori.org

Q. Sun

Channing Laboratory, Department of Medicine,

Brigham and Women's Hospital and Harvard Medical School,

181 Longwood Avenue,

Boston, MA 02115, USA

e-mail: qisun@hsph.harvard.edu

F. B. $\mathrm{Hu}$

Departments of Nutrition and Epidemiology,

Harvard School of Public Health,

665 Huntington Avenue,

Boston, MA, USA

e-mail: fhu@hsph.harvard.edu randomized clinical trials have provided consistent evidence that replacing saturated fat with polyunsaturated fat, but not carbohydrates, is beneficial for coronary heart disease. Therefore, dietary recommendations should emphasize substitution of polyunsaturated fat and minimally processed grains for saturated fat.

Keywords Saturated fat Polyunsaturated fat . Carbohydrate $\cdot$ Monounsaturated fat $\cdot$ Atherosclerosis . Coronary heart disease $\cdot$ Cardiovascular disease $\cdot$ LDL cholesterol - Obesity - Insulin resistance - HDL cholesterol . Triacylglycerol · Triglyceride · Diet · Insulin resistance · Epidemiology $\cdot$ Prospective cohort studies $\cdot$ Randomized controlled trials · Lipids · Weight loss · Nutrient replacement $\cdot$ Clinical studies $\cdot$ Dyslipidemia $\cdot$ Meta-analysis

\section{Introduction}

Dietary saturated fat intake has been shown to increase lowdensity lipoprotein (LDL) cholesterol, and therefore has been associated with increased risk of cardiovascular disease (CVD). This evidence, coupled with inferences from epidemiologic studies and clinical trials, has led to longstanding public health recommendations for limiting saturated fat intake as a means of preventing CVD. However, the relationship between saturated fat and CVD risk remains controversial, due at least in part to the intrinsic limitations of clinical studies that have evaluated this relationship.

Prospective epidemiologic studies have been limited by a reliance on nutritional assessment methods of varying accuracy, the assumption that diets remain the same over the long term, and incomplete adjustments for relevant confounders, including other nutrients. Randomized clinical 
trials testing the effects of reduced saturated fat on CVD risk have generally replaced saturated fat with polyunsaturated fat, monounsaturated fat, or carbohydrate. Therefore, any assumed benefit of reducing saturated fat as gleaned from clinical trial evaluations is complicated by concomitant increases in replacement nutrients.

This article reviews the evidence for adverse effects of saturated fat on lipids and CVD risk and examines the most recent developments that suggest a re-evaluation of the longstanding diet-heart hypothesis (ie, the presumed link of saturated fat with CVD) is timely and necessary. We suggest that the overall evidence from clinical trial and epidemiologic data is most consistent with a protective effect of higher intakes of polyunsaturated fats, with a corresponding increase in the polyunsaturated fat to saturated fat ratio. On the other hand, to the extent that reduction in saturated fat is achieved by increased consumption of refined carbohydrates, there can be a deleterious impact on CVD risk due to adverse effects on components of metabolic syndrome [1].

\section{Effects of Saturated Fat on Plasma Lipids and Lipoproteins}

In humans, saturated fat intake increases LDL cholesterol in comparison with all nutrients except trans fats [2•]. Because saturated fat also increases high-density lipoprotein (HDL) cholesterol, the total cholesterol (TC) to HDL cholesterol ratio (a risk marker for CVD) is not altered. LDL particles are heterogeneous in size, density, and composition. Smaller and denser LDL particles in particular have been strongly associated with atherosclerotic CVD [3]. Changes in dietary saturated fat have been associated with changes in concentrations of larger, more buoyant particles [4]. In the context of a lower-carbohydrate diet ( $26 \%$ of total energy), high saturated fat content $(15 \%$ of energy) provided from dairy products was associated with increased concentrations of large and medium LDL particles, but not small LDL particles, compared with a diet lower in saturated fat ( $8 \%$ of energy) [5].

Studies in animals have shown that saturated fats increase LDL cholesterol by inhibiting LDL receptor activity and enhancing apolipoprotein (apo)B-containing lipoprotein production [6]. This LDL cholesterol-raising effect of saturated fatty acids has been shown to depend on the level of dietary cholesterol, such that the greatest increases in plasma LDL concentrations were observed at the highest levels of dietary cholesterol [6]. In the absence of dietary cholesterol, and when polyunsaturated fatty acid intake is adequate $(5 \%-10 \%$ of energy), saturated fat has been observed to have a negligible effect on LDL clearance in nonhuman primates, and the lipoprotein profile remains relatively normal
(LDL $<90 \mathrm{mg} / \mathrm{dL}$ ) [7]. When individuals or animals are fed excessive calories and dietary cholesterol, specific saturated fatty acids (particularly palmitic acid) can contribute to decreased LDL receptor activity [7]. Nonetheless, LDL receptor downregulation by dietary cholesterol greatly exceeds that by saturated fatty acids [7].

In line with data in humans [8], feeding of dietary monounsaturated fat to nonhuman primates reduced LDL without lowering HDL, and in comparison to saturated and polyunsaturated fat, provided the lowest LDL to HDL ratio [9•]. On the other hand, replacement of some of the saturated fat with monounsaturated fat was associated with an even greater enrichment of LDL particles with cholesteryl oleate, a change in LDL particle composition that has been shown to confer atherogenicity [9•]. However, caution is needed in applying the results from animal experiments to humans.

Different saturated fatty acids have varying effects on LDL cholesterol, HDL cholesterol, and the TC:HDL cholesterol ratio when they replace carbohydrate [10]. The longer chain stearic acid (18:0) has been shown to have no effect on LDL or HDL cholesterol or the TC:HDL cholesterol ratio, and saturated fatty acids of shorter length have been shown to have a greater LDL cholesterol-raising effect, such that lauric acid (12:0) raised LDL cholesterol the most, followed by myristic (14:0) and palmitic (16:0) acids. Lauric acid also increased HDL cholesterol most significantly, and it did this disproportionately to TC, so that its replacement of carbohydrate actually led to a significant decrease in the TC:HDL cholesterol ratio [10].

It has been demonstrated that the expected increases in LDL cholesterol with high saturated fat intake can be attenuated by reductions in levels of small, dense LDL particles resulting from reduced dietary carbohydrate content and/or weight loss $[5,11]$. The influence of weight reduction is illustrated by results of a 12-month study that showed no significant differences in LDL cholesterol change after weight loss among diets with a wide range of saturated fat content [12].

There exists considerable inter-individual variation in the response to dietary saturated fat. The ability of saturated fats to raise LDL cholesterol is enhanced by increased intake of dietary cholesterol as well as baseline LDL cholesterol concentrations [2•]. Intrinsic differences in the regulation of lipid metabolism may partly explain the heterogeneity of responses to dietary saturated fat. In fact, an association of the apoE4 allele with a greater LDL response to saturated fat has been reported in a number of studies, although this effect is confounded by the relationship of apoE4 to baseline LDL levels. Studies have also shown that other factors, including obesity, insulin resistance, hypertriglyceridemia, and female gender, may lead to weaker LDL cholesterol-raising effects of dietary saturated fat [2•].

Finally, the effects of saturated fat can be modulated by the foods in which they are contained [13]. Cheeses may have 
smaller effects on LDL cholesterol concentrations than butter, and fermented dairy foods, such as yogurt, have been associated with LDL reductions [13]. Recently, adipose tissue conjugated linoleic acid (CLA) was found to be inversely associated with risk of myocardial infarction in a case-control study and appeared to offset the strong risk observed with saturated fat [14]. CLA can be found in meaningful concentrations in the milk of pasture-fed cows [14]. However, the potential cardiovascular benefits of CLA need confirmation from prospective cohort studies and intervention trials.

\section{Lipid Effects of Reducing Saturated Fat}

Studies that evaluate the effects of saturated fat on lipids and lipoproteins under weight-stable conditions inherently test the replacement of saturated fat with other nutrients.

\section{Replacement of Saturated Fat with Polyunsaturated Fat}

Replacement of saturated fat with polyunsaturated fat has been shown to decrease TC and LDL cholesterol by lowering LDL cholesterol production rates and/or increasing LDL clearance rates [2•]. Although replacement of saturated fat with polyunsaturated fat has been shown to decrease HDL cholesterol, it decreases LDL cholesterol even more substantially; thus, the HDL:LDL ratio is increased [15] and the TC:HDL cholesterol ratio is decreased [10]. Of note, some studies have suggested that saturated fat may increase LDL cholesterol only if the polyunsaturated fat intake falls below a threshold level; specifically, the availability of linoleic acid may determine the cholesterolemic effects of other fatty acids [16]. The ratio of polyunsaturated fat to saturated fat was recently shown to be inversely associated with LDL cholesterol and TC but not with hemoglobin A1c, blood pressure, serum triglycerides, and HDL cholesterol in 1004 Japanese men and women [17]. Overall, through its effects on plasma lipoproteins (ie, lowering of LDL cholesterol, minimal effect on HDL cholesterol, and lowering of the TC: HDL cholesterol ratio), the replacement of saturated fat with polyunsaturated fat has been projected to modestly lower coronary heart disease risk (by about $10 \%$ for each $5 \%$ energy substitution) [18•]. However, these benefits are likely to be underestimated because polyunsaturated fats can have other benefits beyond cholesterol, including improving insulin sensitivity and reducing inflammation [19].

\section{Replacement of Saturated Fat with Monounsaturated Fat}

Replacement of saturated fat with monounsaturated fat has also been associated with decreased total, LDL, and HDL cholesterol, although the magnitude of reduction for each of these lipids is slightly less than when polyunsaturated fats are the replacement nutrient [15].

\section{Replacement of Saturated Fat with Carbohydrate}

Replacement of saturated fat with carbohydrate results in lower total, LDL, and HDL cholesterol but also increases triglycerides [2•]. The magnitude of reduction of HDL cholesterol is comparable to the reduction in TC, so that the TC:HDL cholesterol ratio is, on average, not changed [10]. Although no effect on plasma lipid levels of exchanging $7 \%$ to $8 \%$ fat for complex carbohydrates was found in a large-scale dietary intervention [20], these null results were most likely attributable to the failure of the study participants to adhere to the study diet [21]. In a study of 52 men and 33 women with baseline dyslipidemia participating in a three-period, 7-week, randomized, crossover dietary intervention study, monounsaturated fat was shown to be the preferred replacement for saturated fat [22]. Although replacement of $7 \%$ saturated fat with both monounsaturated fat and carbohydrate effectively lowered LDL cholesterol, replacement with monounsaturated fat was associated with lesser reductions in HDL cholesterol and lesser increases in lipoprotein(a) and triglyceride concentrations [22].

\section{High Carbohydrate Intake Contributes to Dyslipidemia}

Elevated triglycerides, reduced HDL cholesterol concentrations, and increased concentrations of small, dense LDL particles characterize the dyslipidemia that is part of a metabolic profile considered to be a major contributor to increased CVD risk. Both insulin resistance and high carbohydrate intakes have been shown to contribute to this dyslipidemia, and refined carbohydrates, in particular, can raise triglyceride and lower HDL cholesterol concentrations [23]. Of interest, reductions in dietary carbohydrate, even in the context of a diet high in saturated fat, have been associated with reduced concentrations of small, dense LDL [5].

The type of carbohydrate consumed can affect blood lipid profiles. Using the glycemic index (GI) as a classification system that rates dietary carbohydrates by their ability to increase postprandial blood glucose levels, the consumption of lower-GI foods has been associated with lower triglycerides and higher HDL cholesterol [24]. Substituting low-GI foods for high-GI foods may lower triglyceride concentrations by $15 \%$ to $25 \%$ [25]. There is also considerable evidence that high intake of added sugars [26], and fructose in particular [27], adversely affects all components of atherogenic dyslipidemia. Most recently, an evaluation of data from 6113 adults participating in the National Health and Nutrition Examination Survey (NHANES) showed a positive correlation between added sugars and dyslipidemia [28]; the minimization of sugars as a component of the American diet would be expected to improve these diet-induced abnormal lipid profiles. 


\section{Effects of Saturated Fat on Coronary Heart Disease}

\section{Animal Studies}

Assessment of the effects of dietary saturated fat on atherosclerosis has been studied in various animal models [9•]. Interestingly, in nonhuman cholesterol-fed primates, despite the favorable lipid profiles conferred by monounsaturated fat feeding compared with saturated fat feeding, both diets led to equivalent amounts of atherosclerosis, whereas similar intake of omega- 6 polyunsaturated fat afforded atherosclerosis protection [9•]. Similarly, more atherosclerosis was observed in LDL knockout mice fed monounsaturated fats compared with mice fed either polyunsaturated or saturated fat [29].

\section{Clinical Trials}

Clinical trials designed to evaluate dietary effects on CVD risk have reduced saturated fat by increasing the consumption of a replacement nutrient. A recent meta-analysis of eight clinical studies that replaced saturated fat with polyunsaturated fat $(n=$ 13,614 participants with 1042 CHD events) estimated a coronary heart disease CHD risk reduction of about $10 \%$ for each 5\% energy replacement of saturated fat with polyunsaturated fat, an effect comparable to that predicted from the effects of the intervention on TC:HDL cholesterol ratio [30•].

In contrast, replacement of saturated fat with carbohydrate was not associated with improvements in CHD risk in the Women's Health Initiative, a dietary intervention originally designed to test the effects of a low-fat intervention on cancer and cardiovascular risk [31, 32]. The 8-year study effectively lowered total fat as well as saturated fat intake, although not to the degree that the investigators had intended (the difference in reported fat intake was only $8 \%$ in comparison to the desired $14 \%$ ), thus casting doubt on dietary compliance $[21,31]$. Furthermore, the dietary intervention involved a change in overall dietary pattern, making it difficult to attribute the null effect on CHD risk to changes in a specific nutrient.

\section{Observational and Prospective Epidemiologic Studies}

Although early epidemiologic studies linking saturated fat with blood cholesterol and blood cholesterol with CHD were important in defining a conceptual model of etiology for CVD [33, 34], they were a simplification of the complex relationship of nutrients to disease outcomes. These studies were based on aggregated data and did not adjust for potential confounders (eg, trans fatty acids, polyunsaturated fatty acids, and high-GI carbohydrates), some of which have only more recently come to be known as relevant in modulating CVD risk.
Prospective cohort studies are designed to enable the evaluation of potential baseline disease determinants with future disease outcomes by following a cohort of study participants over a period of time. Large, prospective epidemiologic studies have the advantage of being able to adjust for co-variates, so that the effects of a specific nutrient can be assessed. However, caveats to such studies include the reliance on nutritional assessment methods with varying accuracy and the assumption that diets remain similar over the long term.

Our recent meta-analysis summarized data related to saturated fat and CVD from 21 prospective epidemiologic studies that included 347,747 men and women [35•]. During 5 to 23 years of follow-up, 11,006 people developed CHD or stroke. Intake of saturated fat was not significantly associated with an increased risk of CHD, stroke, or CVD $(\mathrm{RR}=1.07 ; 95 \% \mathrm{CI}, 0.96-1.19 ; P=0.22)$ in adjusted logistic regression models. Because the inclusion of serum cholesterol among the adjusted co-variates may attenuate the relationship of saturated fat with CVD, a subgroup analysis among 15 studies that did not include serum cholesterol in their models was conducted [36-50]. The results from this secondary analysis $(\mathrm{RR}=1.02 ; 95 \% \mathrm{CI}, 0.86-1.19)$ confirmed the lack of association of saturated fat with CVD observed in the larger study. These findings are consistent with another systematic review that showed a nonsignificant association of saturated fat with CVD [51].

The interpretation of these data is complicated by the fact that most epidemiologic studies that were included in the meta-analysis did not explicitly model the effects of nutrient substitution. However, because saturated and total fat are most commonly replaced with carbohydrates, the lack of association between saturated fat and risk of CHD observed in epidemiologic studies can be interpreted as the lack of benefit of substitution of carbohydrates for saturated fat.

Although it has been observed that saturated fat may be associated with CHD or CVD in specific age and gender subgroups [40, 41], the data to support these hypotheses are limited [35•]. There have also been several reports of inverse associations of saturated fat with CVD, specifically, with stroke [50, 52] and coronary atherosclerosis progression in postmenopausal women [53]. However, a significant inverse relationship of saturated fat with stroke was not supported in a meta-analysis of eight such studies [35•]. Finally, although it has been suggested that the association of saturated fat with CHD may be specific to "hard" (ie, fatal) versus "soft" (ie, nonfatal) end points [54], our analysis of the studies that considered fatal CHD as the outcome variable $(n=7)$ yielded a nonsignificant pooled RR estimate of 1.18 (95\% CI, 0.99-1.42) (random effects model; $P$ for heterogeneity $=0.02$; test for overall effect $=$ 0.07). Because the use of fatal CHD as the outcome involves both incidence and survival of the condition, it is 
difficult to separate the role of saturated fat in the development and prognosis of CHD.

\section{Modulation by Replacement Nutrients}

A pooled analysis of 11 cohort studies ( $n=344,696$ persons) used a standardized statistical analysis protocol to explicitly examine replacement effects of individual nutrients. The authors found that replacement of saturated fat with polyunsaturated fat, but not carbohydrate or monounsaturated fat, was associated with decreased risk of CHD [55•]. Indeed, the polyunsaturated fat to saturated fat ratio has been strongly and inversely associated with CHD risk [56]. Of interest, clinical trials that have shown benefit of reducing saturated fat have utilized diets that contained a polyunsaturated fat to saturated fat ratio greater than 0.49 [35•], an apparent threshold above which CHD risk has been reported to be reduced [57].

In contrast, replacement of saturated fat with carbohydrate has been associated with no improvement in CVD risk [57] and a slightly increased risk of nonfatal myocardial infarction [55•]. In addition, a low carbohydrate score (indicative of higher intakes of fat and protein and lower intakes of carbohydrate) as compared with lowfat diets was not associated with increased risk of CHD in women [58].

The type of carbohydrate consumed has been associated with differences in CVD risk. A higher glycemic load, which ranks carbohydrates based on their GI and portion size, was associated with increased risk of CHD in the Nurses' Health Study [58]. More recently, a prospective cohort study in 53,644 women showed an increased risk of myocardial infarction when high-GI foods replaced saturated fats [59•]. Only when saturated fats were replaced with low-GI foods was there an association with a lower risk of myocardial infarction [59•].

\section{Other CVD Risk Factors}

Although beyond the scope of this review article, there are non-lipid CVD risk factors that may be affected by dietary saturated fat. Relevant studies in this regard have recently been reviewed [2•]. Briefly, although a growing body of evidence from animal and cellular studies suggests that dietary saturated fat may reduce insulin sensitivity [60] and promote inflammation, clinical studies have not yielded consistent evidence for such effects, or for adverse effects on blood pressure, thrombosis, and vascular function [2•]. However, there is substantial epidemiologic evidence that higher consumption of omega- 6 polyunsaturated fat at the expense of carbohydrates or saturated fat is associated with lower risk of type 2 diabetes [60].

\section{Conclusions}

Evaluation of the association of saturated fat with lipid profiles and CVD risk requires consideration of the replacement nutrients. Studies in animals and humans support the concept that replacement of saturated fats with polyunsaturated fats results in improved lipid profiles, specifically, decreased TC and LDL cholesterol with minimal decreases in HDL cholesterol, as well as with decreased CVD risk. Although replacement of saturated fats with monounsaturated fats results in improved lipid profiles, the association of this substitution with CVD risk is less clear. Notably, most observational studies did not distinguish between plant and animal sources of monounsaturated fats. Finally, replacement of saturated fat with carbohydrates, particularly refined carbohydrates and added sugars as has occurred over the past few decades, has been associated with dyslipidemia and either no improvement in CVD risk or even increased CVD risk. Given the current epidemics of obesity and insulin resistance, reductions in the consumption of refined carbohydrates and added sugars, in addition to weight control and obesity prevention, should be the prioritized public health dietary goals.

Disclosure Dr. Krauss is a member of the Merck Global Atherosclerosis Advisory Board. He has received grants from the National Dairy Council, National Cattleman's Beef Association, Robert C. and Veronica Atkins Foundation, and the National Institutes of Health. He is the co-inventor of two licensed patents for lipoprotein particle analysis and receives royalties from their use. Dr. Siri-Tarino has received an honorarium and has been supported by a grant from the National Dairy Council. No other potential conflicts of interest relevant to this article were reported.

Open Access This article is distributed under the terms of the Creative Commons Attribution Noncommercial License which permits any noncommercial use, distribution, and reproduction in any medium, provided the original author(s) and source are credited.

\section{References}

Papers of particular interest, published recently, have been highlighted as:

- Of importance

1. Hu FB: Are refined carbohydrates worse than saturated fat? Am J Clin Nutr 2010, 91:1541-1542.

2. - Siri-Tarino PW, Sun Q, Hu FB, Krauss RM: Saturated fat, carbohydrate, and cardiovascular disease. Am J Clin Nutr 2010, 91:502-509. This article provides an updated review of studies evaluating the relationships among saturated fat, blood lipid profiles, and CVD risk.

3. Krauss RM: Lipoprotein subfractions and cardiovascular disease risk. Curr Opin Lipidol 2010, 21:305-311.

4. Dreon DM, Fernstrom HA, Campos H, et al.: Change in dietary saturated fat intake is correlated with change in mass of large 
low-density-lipoprotein particles in men. Am J Clin Nutr 1998, 67:828-836.

5. Krauss RM, Blanche PJ, Rawlings RS, et al.: Separate effects of reduced carbohydrate intake and weight loss on atherogenic dyslipidemia. Am J Clin Nutr 2006, 83:1025-1031; quiz 205.

6. Dietschy JM: Dietary fatty acids and the regulation of plasma low density lipoprotein cholesterol concentrations. J Nutr 1998, $128(2$ Suppl):444S-448S.

7. Hayes KC, Khosla P, Hajri T, Pronczuk A: Saturated fatty acids and LDL receptor modulation in humans and monkeys. Prostaglandins Leukot Essent Fatty Acids 1997, 57:411-418.

8. Mattson FH, Grundy SM: Comparison of effects of dietary saturated, monounsaturated, and polyunsaturated fatty acids on plasma lipids and lipoproteins in man. J Lipid Res 1985, 26:194-202.

9. - Degirolamo C, Shelness GS, Rudel LL: LDL cholesteryl oleate as a predictor for atherosclerosis: evidence from human and animal studies on dietary fat. J Lipid Res 2009, 50(Suppl):S434439. This article reviews the evidence on monounsaturated fats and speaks to the potential adverse effects of monounsaturated fats on CVD risk with evidence provided primarily from animal models.

10. Mensink RP, Zock PL, Kester AD, Katan MB: Effects of dietary fatty acids and carbohydrates on the ratio of serum total to HDL cholesterol and on serum lipids and apolipoproteins: a metaanalysis of 60 controlled trials. Am J Clin Nutr 2003, 77:11461155.

11. Westman EC, Feinman RD, Mavropoulos JC, et al.: Lowcarbohydrate nutrition and metabolism. Am J Clin Nutr 2007, 86:276-284.

12. Gardner CD, Kiazand A, Alhassan S, et al.: Comparison of the Atkins, Zone, Ornish, and LEARN diets for change in weight and related risk factors among overweight premenopausal women: the A TO Z Weight Loss Study: a randomized trial. JAMA 2007, 297:969-977.

13. German JB, Gibson RA, Krauss RM, et al.: A reappraisal of the impact of dairy foods and milk fat on cardiovascular disease risk. Eur J Nutr 2009, 48:191-203.

14. Smit LA, Baylin A, Campos H: Conjugated linoleic acid in adipose tissue and risk of myocardial infarction. Am J Clin Nutr 2010, 92:34-40.

15. Mensink RP, Katan MB: Effect of dietary fatty acids on serum lipids and lipoproteins. A meta-analysis of 27 trials. Arterioscler Thromb 1992, 12:911-919.

16. Wijendran V, Hayes KC: Dietary n-6 and n-3 fatty acid balance and cardiovascular health. Annu Rev Nutr 2004, 24:597-615.

17. Guo Z, Miura K, Turin TC, et al.: Relationship of the polyunsaturated to saturated fatty acid ratio to cardiovascular risk factors and metabolic syndrome in Japanese: the INTERLIPID study. J Atheroscler Thromb 2010 Mar 30 [Epub ahead of print].

18. - Micha R, Mozaffarian D: Saturated fat and cardiometabolic risk factors, coronary heart disease, stroke, and diabetes: a fresh look at the evidence. Lipids 2010 Mar 31 [Epub ahead of print]. This article provides a review of the association of saturated fat with CVD, with a particular emphasis on why and how the nutrient that replaces saturated fat matters.

19. Hu FB, Willett WC: Optimal diets for prevention of coronary heart disease. JAMA 2002, 288:2569-2578.

20. Howard BV, Curb JD, Eaton CB, et al.: Low-fat dietary pattern and lipoprotein risk factors: the Women's Health Initiative Dietary Modification Trial. Am J Clin Nutr 2010, 91:860-874.

21. Willett WC: The WHI joins MRFIT: a revealing look beneath the covers. Am J Clin Nutr 2010, 91:829-830.

22. Berglund L, Lefevre M, Ginsberg HN, et al.: Comparison of monounsaturated fat with carbohydrates as a replacement for saturated fat in subjects with a high metabolic risk profile: studies in the fasting and postprandial states. Am J Clin Nutr 2007, 86:1611-1620.

23. Schaefer EJ, Gleason JA, Dansinger ML: Dietary fructose and glucose differentially affect lipid and glucose homeostasis. J Nutr 2009, 139:1257S-1262S.

24. Barclay AW, Petocz P, McMillan-Price J, et al.: Glycemic index, glycemic load, and chronic disease risk - a meta - analysis of observational studies. Am J Clin Nutr 2008, 87:627-637.

25. Pelkman CL: Effects of the glycemic index of foods on serum concentrations of high-density lipoprotein cholesterol and triglycerides. Curr Atheroscler Rep 2001, 3:456-461.

26. Parks EJ, Hellerstein MK: Carbohydrate-induced hypertriacylglycerolemia: historical perspective and review of biological mechanisms. Am J Clin Nutr 2000, 71:412-433.

27. Stanhope KL, Havel PJ: Fructose consumption: recent results and their potential implications. Ann N Y Acad Sci 2010, 1190:15-24.

28. Welsh JA, Sharma A, Abramson JL, et al.: Caloric sweetener consumption and dyslipidemia among US adults. JAMA 2010, 303:1490-1497.

29. Merkel M, Velez-Carrasco W, Hudgins LC, Breslow JL: Compared with saturated fatty acids, dietary monounsaturated fatty acids and carbohydrates increase atherosclerosis and VLDL cholesterol levels in LDL receptor-deficient, but not apolipoprotein E-deficient, mice. Proc Natl Acad Sci U S A 2001, 98:13294-13299.

30. - Mozaffarian D, Micha R, Wallace S: Effects on coronary heart disease of increasing polyunsaturated fat in place of saturated fat: a systematic review and meta-analysis of randomized controlled trials. PLoS Med 2010, 7:e1000252. This article summarizes the data from randomized controlled trials and shows that replacement of saturated fat with polyunsaturated fat is associated with decreased CVD risk.

31. Howard BV, Van Horn L, Hsia J, et al.: Low-fat dietary pattern and risk of cardiovascular disease: the Women's Health Initiative randomized controlled dietary modification trial. JAMA 2006, 295:655-666

32. Prentice RL, Caan B, Chlebowski RT, et al.: Low-fat dietary pattern and risk of invasive breast cancer: the Women's Health Initiative Randomized Controlled Dietary Modification Trial. JAMA 2006, 295:629-642.

33. Kato H, Tillotson J, Nichaman MZ, et al.: Epidemiologic studies of coronary heart disease and stroke in Japanese men living in Japan, Hawaii and California. Am J Epidemiol 1973, 97:372-385.

34. Keys A, Aravanis C, Blackburn HW, et al.: Epidemiological studies related to coronary heart disease: characteristics of men aged 40-59 in seven countries. Acta Med Scand Suppl 1966, 460:1-392.

35. - Siri-Tarino PW, Sun Q, Hu FB, Krauss RM: Meta-analysis of prospective cohort studies evaluating the association of saturated fat with cardiovascular disease. Am J Clin Nutr 2010, 91:535-546. This meta-analysis of prospective epidemiologic studies showed no association of saturated fat with CVD risk, although the analysis could not determine the optimal replacement nutrient due to the limited data provided by the component studies.

36. Fehily AM, Yarnell JW, Sweetnam PM, Elwood PC: Diet and incident ischaemic heart disease: the Caerphilly Study. Br J Nutr 1993, 69:303-314

37. Ascherio A, Rimm EB, Giovannucci EL, et al.: Dietary fat and risk of coronary heart disease in men: cohort follow up study in the United States. BMJ 1996, 313:84-90.

38. Mann JI, Appleby PN, Key TJ, Thorogood M: Dietary determinants of ischaemic heart disease in health conscious individuals. Heart 1997, 78:450-455.

39. Pietinen $P$, Ascherio A, Korhonen $P$, et al.: Intake of fatty acids and risk of coronary heart disease in a cohort of Finnish men. The 
Alpha-Tocopherol, Beta-Carotene Cancer Prevention Study. Am J Epidemiol 1997, 145:876-887.

40. Boniface DR, Tefft ME: Dietary fats and 16-year coronary heart disease mortality in a cohort of men and women in Great Britain. Eur J Clin Nutr 2002, 56:786-792.

41. Jakobsen MU, Overvad K, Dyerberg J, et al.: Dietary fat and risk of coronary heart disease: possible effect modification by gender and age. Am J Epidemiol 2004, 160:141-149.

42. Leosdottir M, Nilsson PM, Nilsson JA, Berglund G: Cardiovascular event risk in relation to dietary fat intake in middle-aged individuals: data from The Malmo Diet and Cancer Study. Eur J Cardiovasc Prev Rehabil 2007, 14:701-706.

43. Oh K, Hu FB, Manson JE, et al.: Dietary fat intake and risk of coronary heart disease in women: 20 years of follow-up of the Nurses' Health Study. Am J Epidemiol 2005, 161:672-679.

44. Tucker KL, Hallfrisch J, Qiao N, et al.: The combination of high fruit and vegetable and low saturated fat intakes is more protective against mortality in aging men than is either alone: the Baltimore Longitudinal Study of Aging. J Nutr 2005, 135:556-561.

45. Xu J, Eilat-Adar S, Loria C, et al.: Dietary fat intake and risk of coronary heart disease: the Strong Heart Study. Am J Clin Nutr 2006, 84:894-902.

46. Goldbourt U, Yaari S, Medalie JH: Factors predictive of long-term coronary heart disease mortality among 10,059 male Israeli civil servants and municipal employees. A 23-year mortality follow-up in the Israeli Ischemic Heart Disease Study. Cardiology 1993, $82: 100-121$

47. Gillman MW, Cupples LA, Millen BE, et al.: Inverse association of dietary fat with development of ischemic stroke in men. JAMA 1997, 278:2145-2150.

48. Iso H, Stampfer MJ, Manson JE, et al.: Prospective study of fat and protein intake and risk of intraparenchymal hemorrhage in women. Circulation 2001, 103:856-863.

49. He K, Merchant A, Rimm EB, et al.: Dietary fat intake and risk of stroke in male US healthcare professionals: 14 year prospective cohort study. BMJ 2003, 327:777-782.
50. Sauvaget C, Nagano J, Hayashi M, Yamada M: Animal protein, animal fat, and cholesterol intakes and risk of cerebral infarction mortality in the adult health study. Stroke 2004, 35:1531-1537.

51. Mente A, de Koning L, Shannon HS, Anand SS: A systematic review of the evidence supporting a causal link between dietary factors and coronary heart disease. Arch Intern Med 2009, 169:659-669.

52. Iso $\mathrm{H}$, Sato $\mathrm{S}$, Kitamura $\mathrm{A}$, et al.: Fat and protein intakes and risk of intraparenchymal hemorrhage among middle-aged Japanese. Am J Epidemiol 2003, 157:32-39.

53. Mozaffarian D, Rimm EB, Herrington DM: Dietary fats, carbohydrate, and progression of coronary atherosclerosis in postmenopausal women. Am J Clin Nutr 2004, 80:1175-1184.

54. Stamler J: Diet-heart: a problematic revisit. Am J Clin Nutr 2010, 91:497-499.

55. - Jakobsen MU, O’Reilly EJ, Heitmann BL, et al.: Major types of dietary fat and risk of coronary heart disease: a pooled analysis of 11 cohort studies. Am J Clin Nutr 2009, 89:1425-1432. This study provides strong epidemiologic data to support an improvement in CVD risk when saturated fat is replaced with polyunsaturated fat.

56. Hu FB, Stampfer MJ, Manson JE, et al.: Dietary saturated fats and their food sources in relation to the risk of coronary heart disease in women. Am J Clin Nutr 1999, 70:1001-1008.

57. Hu FB, Stampfer MJ, Manson JE, et al.: Dietary fat intake and the risk of coronary heart disease in women. N Engl J Med 1997, 337:1491-1499.

58. Halton TL, Willett WC, Liu S, et al.: Low-carbohydrate-diet score and the risk of coronary heart disease in women. $\mathrm{N}$ Engl $\mathrm{J}$ Med 2006, 355:1991-2002.

59. - Jakobsen MU, Dethlefsen C, Joensen AM, et al.: Intake of carbohydrates compared with intake of saturated fatty acids and risk of myocardial infarction: importance of the glycemic index. Am J Clin Nutr 2010, 91:1764-1768. This is one of the first studies to document a difference in the risk associated with CHD of carbohydrates of varying quality.

60. Riserus U, Willett WC, Hu FB: Dietary fats and prevention of type 2 diabetes. Prog Lipid Res 2009, 48:44-51. 